EPJ Web of Conferences 21, 10004 (2012)

DOI: $10.1051 /$ epjconf/20122110004

(C) Owned by the authors, published by EDP Sciences, 2012

\title{
Spatial- and Time-Correlated Detection of Fission Fragments
}

\author{
C. Granja ${ }^{1, a}$, V. Kraus ${ }^{1}$, Y. Kopatch ${ }^{2}$, S.A. Telezhnikov ${ }^{2}$, J. Vacik ${ }^{3}$, I. Tomandl ${ }^{3}$, M. Platkevic ${ }^{1}$, and \\ S. Pospisil ${ }^{1}$ \\ ${ }^{1}$ Institute of Experimental and Applied Physics, Czech Technical University in Prague, Czech \\ Republic \\ ${ }^{2}$ Joint Institute for Nuclear Research (JINR), Dubna, Russia \\ ${ }^{3}$ Nuclear Physics Institute, Ac. of Sciences of the Czech Republic, Rez near Prague, Czech Republic
}

\begin{abstract}
With the goal to measure angular correlations of fission fragments in rare fission decay (e.g. ternary and quaternary fission), a multi-detector coincidence system based on two and up to four position sensitive pixel detectors Timepix has been built. In addition to the high granularity, wide dynamic range and per pixel signal threshold, these devices are equipped with per pixel energy and time sensitivity providing more information (position, energy, time), enhances particle-type identification and selectivity of event-by-event detection. Operation of the device with the integrated USB 2.0 based readout interface FITPix and the control and data acquisition software tool Pixelman enables online visualization and flexible/adjustable operation for a different type of experiments. Spatially correlated fission fragments can be thus registered in coincidence. Similarly triggered measurements are performed using an integrated spectrometric module with analogue signal chain electronics. The current status of development together with demonstration of the technique with a ${ }^{252} \mathrm{Cf}$ source is presented.
\end{abstract}

\section{Motivation}

Charged-particle coincidence correlated measurements such as angular correlations between rare and main fission fragments measured with conventional detectors provide only partial and limited information (energy cutoff, narrow range of studied ion $\mathrm{Z}$ numbers). Many of these drawbacks arise from the standard solid state detectors used so far which can be solved simultaneously by usage of highly segmented single-quantum counting pixel detectors.

Rare fission decay with the emission of one (ternary fission) or two (quaternary fission) light mass rare charged particles - or rare fission fragments is a valuable tool to explore the dynamics and structure of atomic nuclei by probing the fissioning system near the scission point and the fissioning reaction and decay mechanisms. Spectrometric measurement of the characteristics of fission products is the necessary input for the understanding of fission configurations and dynamics of reaction mechanisms in the final stage of the fissioning process. Observables such as the angular momenta of RFF correlated with fission fragments are closely related to the dynamics of the fissioning system in its final stages [1],[2]. When passing the barrier the fissioning system can probe different channels as the rare fission fragments are produced at the moment of scission in the neck region of the nascent fission fragments [1]. While being a rather rare process, these light charged particles serve as a probe of the nucleus at a time close to scission and while are considered to emerge from the neck of the fissioning nucleus much earlier than scission of the nucleus into the

a e-mail : carlos.granja@utef.cvut.cz

This is an Open Access article distributed under the terms of the Creative Commons Attribution-Noncommercial License 3.0, which permits unrestricted use, distribution, and reproduction in any noncommercial medium, provided the original work is properly cited. 
heavy main fission fragments occurs. While most rare fragments are emitted almost perpendicularly with respect to the fission axis (equatorial emission), Rare fragments may be emitted also along the axis of fission (polar emission) [3].

Ternary fission has been widely studied experimentally by measurements of the energy spectra and fractional yields of the rare fragments or by experiments on the angular and energy correlations between the rare and the binary fragments - e.g. Ref [4]. Due to technical constraints of conventional detectors so far charged-particle coincidence measurements such as angular correlations between rare and main fission fragments [5] could only be measured under certain circumstances (energy cutoff, low-Z). Many of these limitations arise from the standard solid state detectors used so far which we propose can be overcome simultaneously by using highly segmented single-quantum counting pixel detectors.

\section{Experimental}

\subsection{Hybrid semiconductor pixel detector}

The quantum counting pixel detector Timepix [6], developed within the family of hybrid semiconductor pixel detectors of the Medipix type [7], is equipped with energy and time sensitivity capability per pixel providing not only high granularity and per pixel threshold but also per pixel sensitivity and wide dynamic range (energy,time). Timepix provides the possibility to measure detection times and/or energy deposition in each individual pixel. This is realized by the ability of the detector to operate the pixels in one of the different modes: (i) Counting mode, where each pixel counts the number of events/signals crossing the threshold, (ii) Time-Over-Threshold (TOT) mode, where the energy deposited per-pixel is measured, and (iii) Timepix mode, where the time of interaction per-pixel is registered. All pixels are configurable and each pixel can be independently/separately configured to different mode enabling different pixels to run under different modes for the same measurement. Timepix provides multi-parameter event-by-event spectroscopic information (position, energy and time) of basically all types of charged particles in a wide energy range (pixel energy threshold $\approx 4 \mathrm{keV}$ ).

The response of the pixel detectors of the Medipix type such as the Medipix2 device [8] for detection of heavy ions and fission fragments has been examined [9]. The spectroscopic response of the Timepix device has been extended and characterized for heavy ions in the $10-110 \mathrm{MeV}$ region $[10]$.

\subsection{Detection of fission fragments}

In addition to the intrinsic high spatial granularity of the pixel detectors of the Medipix type, the Timepix device allows applying timing and spectral correlated techniques in the same sensor for enhanced background suppression and unambiguous event-by-event detection [11]. Control, power and DAQ of the pixel detectors are driven and managed by the software package tool Pixelman [12]. We can for example follow and visualize the life history of single nuclei implanted in the same sensor [11], [13]. The resolving power and event-by-event detection of fission fragments and charged particles in a single detector have been investigated [14]. The response of the detector to an alpha and fission source is shown in Figure 1.

\subsection{Correlated detection by two or four detectors}

Several hardware techniques setups have been developed and tested [15] including self triggering by independent analog chain analysis with integrated spectrometric module and synchronized readout on various setups using two and four detectors. This self trigger is taken from the common sensor 

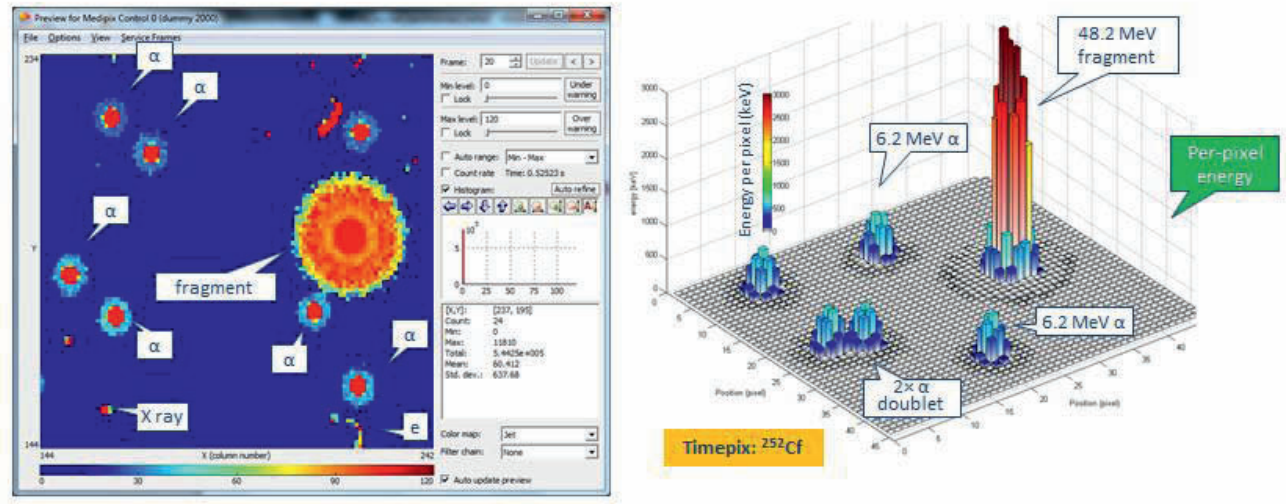

Fig. 1. Detection of a fission fragment and alpha particles from a spontaneous fission source ${ }^{252} \mathrm{Cf}$ by the pixel detector Timepix. Only a portion of the sensor area is shown of about $40 \times 40$ pixels. In addition to the spatial (2D) information the energy registered per pixel is recorded and can be shown as a third axis by the vertical bar in color. Pile-ups as well as unwanted background such as X-rays and fast electrons are resolved.

back-side-pulse signal. This signal can be, like for standard p-i-n diodes, used as trigger above a selectable signal amplitude threshold. In this technique we employ a custom made spectrometric module integrated into a single PCB board which can be attached directly to the readout interface (USB 1.0) of the pixel detector. This module is operated with a Windows running plugin application which loads and controls the sensor bias as well as the readout DAQ.

\section{Spatial- and Time-correlated detection}

In order to detect particles in coincidence, such as the two binary fission fragments or a rare fission fragment correlated to the main fragments, respectively, we prepared a setup with several pixel detectors. Results of correlated detection of pairs of binary fragments are shown in Figure 2 with many assignments of spatial- and time-correlated events. In this setup, the Timepix detectors are operated in time mode which allows registering the time record of individual pixels during the given exposition time. Thus the time record of all events can be traced back and can be correlated among different detectors. This possibility has been tested with data measured for up to four detectors.

\section{Conclusions}

The operation of two and more pixel detectors Timepix and Medipix 2 for spatial- and temporalcorrelated detection of single events as well as for coincidence operation for detection of two and three particles have been achieved for spatially correlated studies of fission. Several techniques have been constructed and tested under a number of particular detector and experiment conditions such as pixel threshold, sensor bias, pixel signal acquisition time (IKRUM parameter) and measurement exposure time. We have gradually developed the spatial- and time-correlated technique in the same sensor enabled by the per-pixel energy/time sensitive Timepix device. We developed and tested modes of self trigger in the same detectors. Synchronized data acquisition and readout are possible by a custom made arbiter control unit. Analysis of angular distributions/correlations as well as data evaluation of ternary fission are underway. Long measurements are planned for studies of quaternary fission. 

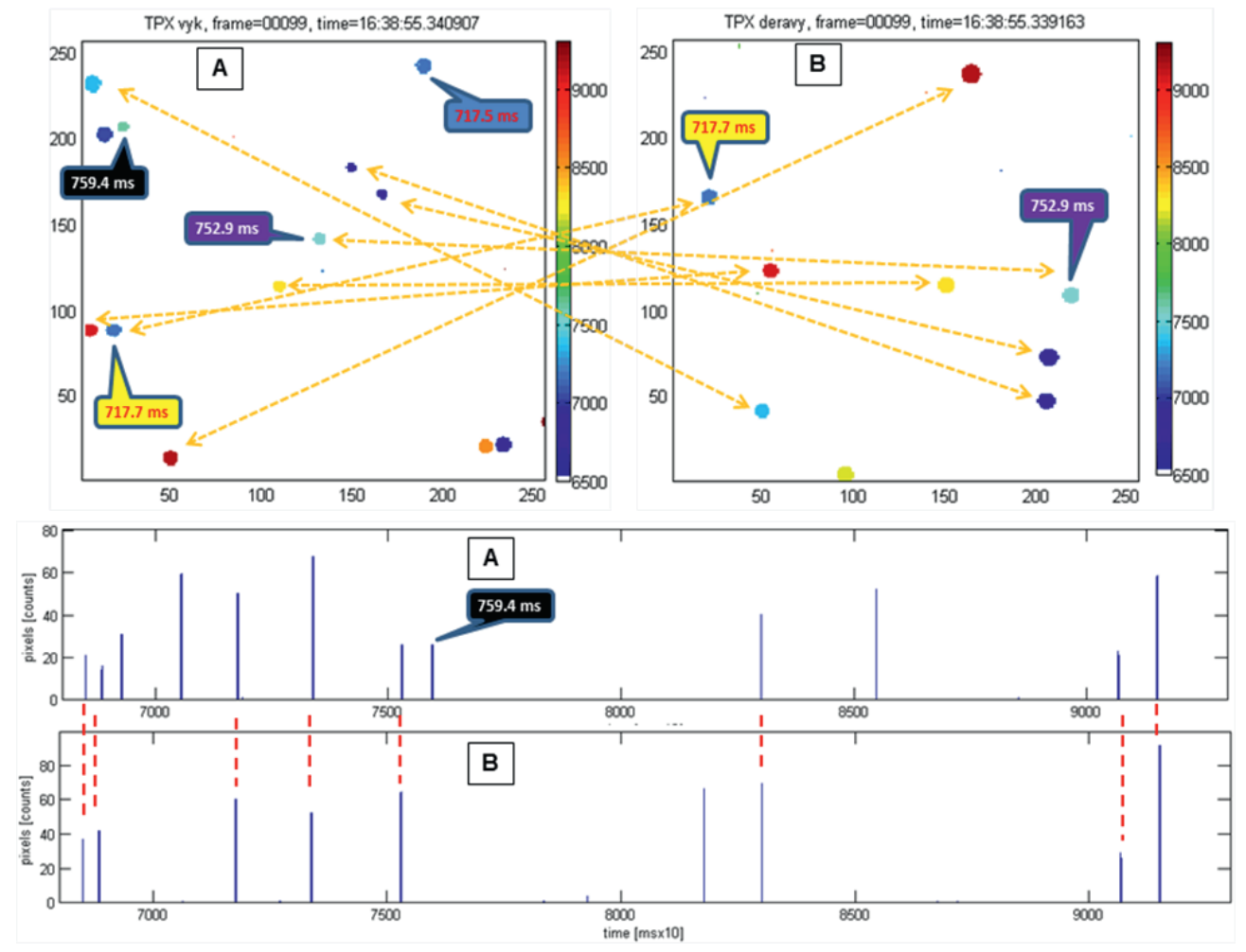

Fig. 2. Detection in coincidence of two fission fragments by two Timepix pixel detectors operated in high threshold and long exposure time $(300 \mathrm{~ms})$. The shutter open was driven by one of the detectors (arbitrary shutter open time). The spatial information, given by the independent per-pixel response and the detector high spatial granularity of the full $256 \times 256$ pixel matrix of each detector (top), is correlated to the temporal information (bottom) provided by the operation of all pixels in time (i.e. Timepix) mode registering the events interaction time. Correlated events are linked by straight arrows for clarity. The pixel thresholds are set high thus suppressing and ultimately removing the signals of alpha particles and allowing for long exposure times (here $300 \mathrm{~ms}$ ).

\section{References}

1. C. Wagemans, The nuclear fission process, CRC Press, USA, (1991)

2. F. Gonnenwein, A. Moller, M. Croni, et al., Nuovo Cimento della sociata italiana di fisica A nuclei particles and fields, 110, 1089-1095 (1997)

3. L. Nowicki, E. Piasecki, J. Sobolewski, et al., Nuclear Physics A 375, 187-216 (1982)

4. Yu. N. Kopatch, M. Mutterer, D. Schwalm, P. Thirolf, and F. Gönnenwein, Phys. Rev. C 65, 044614 (2002)

5. M. Jandel, J. Kliman, L. Krupa, et al., Eur. Phys. J. A 24, 373-378 (2005)

6. X. Llopart, R. Ballabriga, M. Campbell, and W. Wong, Nucl. Inst. and Methods in Phys. Res. A 585, 106-108 (2007)

7. Medipix Collaboration - see http://medipix.cern.ch

8. X. Llopart, M. Campbell, R. Dinapoli, D. San Segundo, E. Pernigotti, IEEE Trans. Nuclear Sciences, No. NS-49, 2279 (2002)

9. C. Granja, Z. Vykydal, Y. Kopatch, J. Jakubek, S. Pospíšil, and S. A. Telezhnikov, Nucl. Inst. and Methods in Phys. Res. A 574, 472-478 (2007) 


\section{$\mathrm{CNR} * 11$}

10. C. Granja, J. Jakubek, U. Köster, M. Platkevic, S. Pospisil, Nucl. Inst. and Methods in Phys. Res. A 633, S198-S202 (2011)

11. J. Jakůbek, M. Platkevič, C. Granja, U. Koester, S. Pospíšil, Nucl. Inst. and Methods in Phys. Res. A 633, S203-S205 (2011)

12. T. Holy, J. Jakubek, S. Pospisil, J. Uher, D. Vavrik, and Z. Vykydal, Nucl. Inst. and Methods in Phys. Res. A 563, 254 (2006)

13. C. Granja, J. Jakůbek, U. Koester, M. Platkevič, S. Pospíšil, Amer. Insti. of Physics (AIP) Conference Proceedings Series, No. 1265, 497-500 (2010)

14. C. Granja, J. Jakubek, S. Pospisil, Z. Vykydal, Y. Kopatch, S. Telezhnikov, Nuclear Science Symposium IEEE NSS/MIC, Conf. Record, Dresden, 934-935 (2009)

15. C. Granja, V. Kraus, J. Jakubek, S. Pospisil, P. Masek, Z. Vykydal, M. Platkevic, Z. Kohout, Y. Kopatch, S.A. Telezhnikov, Nuclear Science Symposium IEEE NSS/MIC, Conf. Record, Knoxville, 1578-1584 (2010) 